\title{
On the use of a controlling database for Cognitive Radio in Wireless Body Area Networks
}

\author{
Gintarè Sukarevičienè \\ Department of Applied informatics \\ Vytautas Magnus University \\ Vileikos 8, LT-44404, Kaunas, \\ Lithuania \\ +37060568925 \\ g.sukareviciene@gmail.com
}

\author{
Dainius Jankūnas \\ Department of Applied informatics \\ Vytautas Magnus University \\ Vileikos 8, LT-44404, Kaunas, \\ Lithuania \\ +37062908917 \\ dainiusneo@gmail.com
}

\author{
Vladislav V. Fomin \\ Department of Applied informatics \\ Vytautas Magnus University \\ Vileikos 8, LT-44404, Kaunas, \\ Lithuania \\ +37065562473 \\ vvfomin@gmail.com
}

\begin{abstract}
Cognitive Radio (CR) is a fast growing technology domain, which is currently in the stage of transitioning from R\&D labs through international standardization committees and fora to the market. One particular promising application domain for $\mathrm{CR}$ is the so called Wireless Body Area Networks (WBAN). This domain is concerned with small-scale radio networks in general, and use of CR technologies in hospital environment in particular. It is expected that it can eventually lead to technologies not just for health care facilities, but also for in-home use and much more.

Already with extant technology it is possible to create miniature wireless sensor devices to be placed on human body for monitoring and analysis purposes. What has not been solved yet, is the way various WBAN sensors would communicate with each other without creating interference.

Recent advancements in CRS saw developments of control mechanisms for different applications, especially for larger scale (city/regional/national) uses of vacating TV broadcasting spectrum, based on utilization of Geolocation Database (GDB) as communication control registry and visualization tool. In this paper we propose technical characteristics for a controlling database (DB) to allocate radio resources and to be deployed in a hospital environment for WBAN, thus contributing to the ongoing development in CR domain.
\end{abstract}

\section{Categories and Subject Descriptors}

H.2.8. [Database management]: Database applications: Spatial databases and GIS.

\section{General Terms}

Management, Performance, Economics, Human Factors, Standardization.

\section{Keywords}

Cognitive Radio, Body Area Networks (BAN), controlling database for WBAN, hospital bandwidth, mHealth devices.

\section{INTRODUCTION}

Today wireless technology is rapidly evolving and playing a significant role in offering wide range of new opportunities that lead to more dynamic, "on-the-go" environment, enhanced productivity and much more. It is likely that wireless systems can bring plenty of benefits in the Body Area Networks (BAN), which is emerging as promising domain for Cognitive Radio Systems (CRS) with applications being developed for such sectors as healthcare, surveillance, entertainment and other. It is the first two sectors that we will focus on in this paper. The emergence of new wireless systems often requires a separate radio channel or even several of them for data transferring, to accommodate the growing volume of data transfers. Thus, the wireless spectrum crunch becomes an important issue for wireless Medical devices (mHealth devices), which require consistent and reliable access to wireless signal in order to be safe and effective [5].

Cognitive Radio (CR) is a promising solution for this issue, as it allows maximizing utility of radio spectrum by interacting with surrounding radio environment, learning about the environment, and using the learned knowledge to improve communication [2]. Vice versa, sharing of spectrum by CR would make it difficult to detect excessive sources of interference. As a result, there is the need for a controlling entity - a controlling database (DB) to be deployed in a hospital environment for Wireless Body Area Networks (WBAN).

On May $24^{\text {th }}, 2012$ the FCC dedicated spectrum (for both licensed and unlicensed use, previously used almost exclusively by commercial test pilots) enabling Medical Body Area Networks (MBAN) to transform patient care, lower health care costs, and spur wireless Medical innovation [7].

However, to date there are no communication protocols or controlling DB characteristics developed at the international level. In this work, aiming to extend the conceptual description of controlling DBs for different applications areas [10], we suggest the technical characteristics for a controlling DB for the application of Body Area Network (BAN) wireless sensors (WS) using the $\mathrm{CR}$ communication paradigm.

This paper will be organized as follows: section 2 introduces the existing works on controlling DB and seeks answering such questions as, why a DB is needed and what applications do we know. In section 3, the paper proposes specifics of the hospital environment and WBAN. Specifics will be based on scenarios as 
we know (cardiology, gynecology), proposed DB structure and DB characteristics as we develop. Conclusions will be provided in section 4

\section{THE NEED FOR A CONTROLLING DATABASE}

An experimental Geolocation Database (GDB) was created and tested for TV White Space (TVWS) usage for regular devices and microphones in Lithuania [8]. As for now, it is necessary to give a short reasoning for why a controlling DB for WBAN can also be a useful solution.

First of all, WBANs offer a cost-effective, life saving applications for improving patient care and saving lives. For example, WBANs integrated with GPS should allow dispatchers to know the exact location of patients in distress. This would provide long-term continuous monitoring of vital signs for high-risk cardiovascular patients [11]. The specifics are that wireless sensors are implanted in some clothes which have GPS and send information wirelessly to a local device, e.g., a computer or a mobile phone, and this device using internet connection sends alarms to dispatch. However, to date, even in such technologically advanced countries as US approximately $50 \%$ of hospital patients are not monitored [9].

In Lithuania, there are plenty of hospitals, of which Vilnius University Hospital "Santariškių Klinikos" and Kaunas University Hospital "Kauno Medicinos Universiteto Klinikos" are the most popular ones [3]. It is the main key source in which the Medical innovation can be primarily fitted. Our interviews with several doctors show that there are no wireless sensors used in hospitals in Lithuania, while application of such would be welcomed in at least two broad departments - cardiology and gynecology.

Besides the opportunities above, the concept of Medical innovation requires optimal spectrum utilization. When we talk about limited spectrum and huge number of wireless Medical devices (mHealth devices), the problem arises of how that spectrum should be allocated and used at any given time and location. If this spectrum is not optimally utilized there will be situations when wireless sensors will not work due to inefficient frequencies allocation. According the FCC rules, every device is required to periodically query the spectrum database instead of performing spectrum sensing [4]. In hospitals it becomes very important because every device must work anytime and anywhere. This means that CR needs a controlling entity, which can help monitor spectrum for interference and to identify available channels and frequencies as well.

Looking at TVWS applications it is proved that DB is the most suitable controlling entity to use the radio spectrum in the most efficient, flexible and productive way. Static spectrum sharing is the historical norm, now we need to make it dynamic. The DB must contain all information about mHealth devices being used at any channel, and calculate available channel information based on propagation model. DB must know which channels could be allocated to new mHelath devices if needed as well as DB must support mobility because mHealth devices query the DB whenever they move more than 100 meters away from their current locations.

The DB is easy to access if there is a well placed infrastructure. So there are key criteria of such DB: channel allocation engine, public access, conversion routines and protected entity registration. The only thing we need is CR devices (so-called
White Space Devices (WSDs), that could transmit and receive across channels in very wide range of frequencies. In other words, WSDs should have ability to set transmit and receive frequencies in response to external information. The cost of such Cognitive Radios combined with free DB would be low, compared with devices that have spectrum sensing ability. Thus, application of CR for WBAN could reduce the burden of resources constraints of mHealth devices working on batteries, improve spectrum utilization, and free patients from the set of wires.

\section{SPECIFICS OF THE HOSPITAL ENVIRONMENT AND WBAN}

Wireless Medical applications will require a controlling DB to fit their demands. There can be quite some variation in how a DB can meet these demands of different applications. This variation can lead to different DB characteristics, of which we have defined the most important ones as follows: allowed degradation, frequency of DB updating, channels reservation, location accuracy, harmonized level, operating bandwidth, synchronization, transmit power, processing power, costs, ownership of DB [10].

First of all, allowed degradation is caused by wireless signals interference within a specific frequency band. In order to reduce the level of degradation close to zero percent a geolocation database is needed where devices can determine their location and get the list of channels they can use in that location. That GDB would be a controller for assigning channels for those devices.

Secondly, the controlling DB should be able to update rapidly because of frequently changing hospital consumers as well as the spectral occupancy information. New devices should be registered immediately and this process should be automated.

Channels reservation is not allowed because devices will be registering automatically due to time saving when patient comes to the doctor. No human intervention is needed. It is not convenient if the doctor has to register all devices on patient's body (e.g. the number of such devices can be from 1 to a dozen or more). There should be different levels of priority of one device over another. For example, cardiology-related devices for monitoring human life under emergency conditions may be given priority over devices for regular check-ups.

When we talk about BAN the concept of location accuracy has different understanding in GDB context. The system will be showing the human body and the locations of wireless sensors on it, instead of showing a geographical city map with GPS coordinates. So a DB will show "anatomical" instead of "geographical" features. Imagine there is a human body with 30 sensors attached to it, and the doctor removed 28, and forgot about the remaining 2 . The system can warn and help locate the remaining sensors.

As there can be private and public hospitals in a country, each of them may develop their own system, but a national standard is preferable, so they should be harmonized in the national level. For example, a patient has attached sensors of private hospital which uses its own DB, and these sensors do not work in public hospital with another DB because of different DB structure and sensors in his body can not communicate. That is the main reason why all DBs in national hospitals should use the same structure to be able to communicate with all sensors.

The unlicensed nature of ultra wideband (UWB) spectrum makes it essential for a UWB device to coexist with other devices that 
share the same spectrum [1]. There are two main competing technical approaches to the development of the UWB systems as the radio interface of the WBAN. These two approaches are Impulse Radio (IR Radio) and Multi Band OFDM (MB OFDM). The MB-OFDM approach has been primarily used for applications such as streaming video and wireless USB with data rates of $480 \mathrm{Mb} / \mathrm{s}$, while IR-UWB radios, however, can be designed with relatively low-complexity and low power consumption [6]. Compared to single-band multiple-band UWB can maintain associated the power level, but data throughput can be significantly increased, i.e., from approximately $55 \mathrm{Mbps}$ to $450 \mathrm{Mbps}$ over distances up to $10 \mathrm{~m}$. This means that multipleband operation gives better bandwidth as compared to the singleband if we think about speed of transferring data between sensors and central devices. Multiband OFDM can comply with local regulations by dynamically turning off certain tones or channels in software [1].

When we talk about synchronization we need to think about the architecture of DBs - are all devices connected to a single DB, or are there different DBs which must be synchronized. If sensors' battery level goes down, or if the body is moved to a different location (room), DB may have to issue alert on the "out of range" situation. If it happens, other sensors now can try to use channels of "out of range" sensors. If there is more than one DB, synchronization would be required in order to free more channels. Here we can also think of "roaming" feature - if a body is moved from one room to another, the sensors may reconnect to a different device, which is connected to a different DB. In that case the synchronization is required to know the sensor's information. The synchronization can be made between local DBs when each department is using its own DB. But if we talk about rapid synchronizations we should evaluate that this operation can take some time and resources if there are many records. So synchronization should be used only if changes in DB are slow.

A DB needs to know where devices are located, the frequencies they are using, power levels at those frequencies and the signal strength of those devices. Knowing such information DB can determine the maximum transmit power for the device without causing interference. This is very important to set maximum power levels of the devices that they could not interfere with each other.

It is also important to know that the more nodes (end devices/wireless sensors) there are, the more queries the DB will get. If a DB needs to inform a lot of devices/nodes, it will need a lot of processing power.

Spectrum sensing requires cognitive devices that could "sense" usage by monitoring the spectrum for activity, but currently it is expensive to use. So in the case of costs, a DB is the cheapest option in fact that there are free open source DBs (i.e. MySQL). Furthermore, the cost in terms of transmission overhead for updating and retrieving information entries from the database to the sensor node or sink node may become a burden on extremely simple resource constraint devices working on batteries. WSDs do not need to have technology to scan spectrum for used channels, it is enough to connect a DB and get the list of possible channels in that location.

Finally, the ownership of the DB will likely be the hospital itself. But the structure of DB should be determined by the national regulator.
To show that Medical applications require these DB capabilities, this paper will apply a controlling DB characteristics above for wireless heart monitoring application:

- Allowed degradation: it is critical application (no degradation allowed/otherwise people might die);

- Frequency of updating: should be very rapid (again, because of the critical nature of the application);

- Channels reservation: the heart monitor should be "always on", so registration is not feasible;

- Location accuracy: requires high location accuracy, e. g. up to $1 \mathrm{~m}$ accurate (as this is a "local"/"static" application);

- Harmonized level: DB should allow this application to have unified access to the DB in all countries of the harmonized region, at least within the certain national region;

- Operating bandwidth: should only look at single band (this application is not looking for "opportunistic" ways of finding new spectrum. It just wants to protect itself);

- Synchronization: it should be useful if there is the need to synchronize records obtained through registration procedures with other hospitals DBs;

- Transmit power: devices can be portable or fixed. Multiple sub-bands compared to single-bands UWB can maintain associated the power level, but data throughput can be significantly increased.

- Processing power: there is limited amount of nodes (maybe 300 in the room?), so low processing power of DB is needed.

- Costs: the more cheaper application is the more usable it will be;

- Ownership of DB: public.

\subsection{The database requirements for WBAN}

Specific requirements are also needed to a controlling $\mathrm{DB}$ for WBAN. Here FCC guidelines for TVWS GDB can be used as a departure point:

1. A DB must receive requests for lists of available channels from WBAN devices, identify the type of device (portable or fixed) that is making the request, and provide a correct and accurate list of available channels to the device;

2. A DB must have the capability to register venues at which wireless sensors are used;

3. A DB must control for the number of active sensors if there are limit set in the maximum number of wireless sensors;

4. A DB must have capability to prior devices by relevance;

5. A DB must set the correct power level that can be used by wireless sensor without causing interference;

6. Synchronization of records obtained through registration procedures;

7. A DB administrator must be able to provide to the Regulator, upon request, any information contained in the DB. The requested information can be anything from a full copy of all the records in the DB to specific information contained in a single record. A DB administrator must also be able to make available audit trail information (which devices requested access at specific times and locations). A DB administrator must retain sensors and channel information for 30 days for possible audits;

8. Implementation of enforcement actions:

- a DB administrator is required to remove any information from the DB upon direction of the Regulator;

- a DB must be capable of indicating, upon request of the Regulator, that no channels are available when queried by a specific sensor or model of it. We also need this capability to be implemented such that a model of sensor or all sensors can be 
denied channels on the human body area defined by a point and radius or the area within a set of vertices.

9. A DB must have the ability to verify that a sensors serial number and location are unique to detect "spoofing" of devices and to deny service when spoofing is detected.

10. A DB must only provide channel availability service to certified WBAN devices and must have procedures and features to ensure that the $\mathrm{DB}$, its contents and its operations cannot be compromised by outside parties.

\subsection{Database structure}

Based on the proposed characteristics, the DB fields can be set to store the follows data: location, channel, the device model or type, the level of priority, time when replace or charge battery.

Much like that controlling DB for WBAN must have field for location. This could be the case for DB consulted by mHealth device about sensors position on human body (e. g. in arm, chest or head).

Such DBs may also need to be able to specify channel assigned to wireless sensor.

Furthermore, there might also be some variation with regard to the device model or type - it will be portable or fixed.

Another field of DB may be for the information about the level of priority. DB for WBAN need to be able to set priority of wireless sensor, in extra case low priority devices will be automatically disconnected and freed channels assigned to new sensors.

Finally, DB must calculated battery level and the remaining time left to battery change in order let the doctor know when to replace battery. This means that time when replace or charge battery should also be included in the controlling DB structure.

As well as there can be discussion on how that DB would look like. Will there be one DB for all mHealth devices solutions? Or will there be separated DBs for every mHealth device, accessible from the primary station based on the collaborative data?

\section{CONCLUSIONS}

Despite the fact that spectrum sensing and allocation based on "localization" information of the physical location of the sensor nodes, "mobility" of the patient, and certain "Quality of Services" metric will become a complex optimization issue, field interviews and common knowledge suggest there is a great facility to use CR with the aid of controlling DB for WBAN. The controlling DB should fulfill the needs of Medical applications in the case to use benefits that they offer. The technical characteristics proposed in this paper are inspired by the proposed characteristics to the GDB in the previous analysis that clearly demonstrated that the different GDB characteristics required serving a wide range of wireless applications [10].

In our future research an experimental facility will be set up to test drive a DB with the proposed characteristics. This will be done in collaboration with colleagues developing communication protocol for WBAN mHealth WSDs.

\section{ACKNOWLEDGMENTS}

We are thankful to our colleagues from COST Action IC0905 "COST-TERRA" for sharing their ideas and support in developing this paper.

\section{REFERENCES}

[1] Batra, A., et al. 2003. Multiband OFDM: Why it Wins for UWB. http://www.eetimes.com/design/communicationsdesign/4008964/Multiband-OFDM-Why-it-Wins-for-UWB.

[2] Arslan, H., Ahmed, S. 2007. Applications of Cognitive Radio, Cognitive Radio, Software Defined Radio, and Adaptive Wireless Systems. Springer.

[3] CCHS - CSIC. 2010. Catalogue of World Hospitals: Hospitals of Lithuania. http://hospitals.webometrics.info/rank_by_country.asp?count ry $=1$ t.

[4] FCC. 2010. Second memorandum opinion and order. http://hraunfoss.fcc.gov/edocs_public/attachmatch/FCC-10174A1.pdf.

[5] FCC. 2012. George Washington University Hospital, Hospital Auditorium, Lower Level. http://www.fcc.gov/events/genachowski-remarks-unleashingspectrum-medical-body-area-networks.

[6] Fernandes, J. R. and Wentzloff, D. D. 2010. Recent advances in IR-UWB transceivers: An overview, in 'ISCAS', IEEE. 3284-3287.

[7] Grace, N. 2012. Unofficial announcement of Commission action. http://www.fcc.gov/document/fcc-dedicatesspectrum-enabling-medical-body-area-networks. FCC, News Media Information 202/418-0500.

[8] Jankūnas, D. Experimental site to showcase the development of the national WS GDB for Lithuania. http://www.costterra.org/WSDB/map/.

[9] Mharmer, 2012. FCC Opens Up Wireless Spectrum for Medical Body Area Networks ("MBANs"). http://blog.modernmobileapps.com/post/2012/06/15/FCCOpens-Up-Wireless-Spectrum-for-Medical-Body-AreaNetworks-\%28MBANs\%29.aspx.

[10] Sukarevičienè, G., Barrie, M., Delaere, S., Ballon, P., Fomin, V. 2012. Identifying Geolocation database requirements for distinct wireless applications. Working paper.

[11] Zheng, J.W., Zhang, Z.B., Wu, T.H. and Zhang, Y. 2007. A wearable mobihealth care system supporting real-time diagnosis and alarm. Med. Bio. Eng. Comput. v45. 877-885. 